\title{
Epidural hematoma unmasked after a contralateral craniectomy for a depressed skull fracture
}

\author{
Younes Aissaoui, MD • Ayoub Belhadj, MD
}

Received: 16 December 2014/ Accepted: 19 January 2015 / Published online: 27 January 2015

(C) Canadian Anesthesiologists' Society 2015

A 17-yr-old healthy male suffered severe head trauma (Glasgow Coma Scale 7/15) following a road traffic accident. The initial computed tomography (CT) scan of the patient's head showed a left parietotemporal depressed skull fracture with mass effect and signs of left hemispheric brain edema (Figure A). The patient was brought to the operating room for surgical repair of the fracture, and intraoperatively, we encountered significant brain swelling after removal of bone fragments. Osmotherapy was implemented with an intravenous infusion of $20 \%$

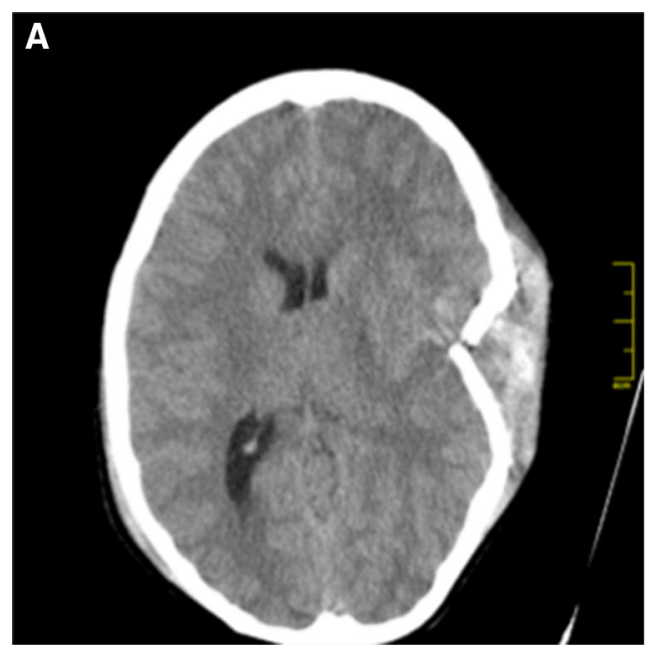

Figure Computed tomography (CT) scan (A) demonstrating a left parietotemporal depressed skull fracture with mass effect and signs of left hemispheric brain edema. The postoperative CT scan (B) mannitol $50 \mathrm{~g}$; a duraplasty was performed, and the bone flap was not put back in place. At the end of the procedure, the patient's right pupil was fixed and dilated, and an immediate postoperative CT scan showed a large right frontotemporal epidural hematoma $(\mathrm{EDH})$ with signs of diffuse intracranial hypertension and midbrain compression (Figure B). The patient underwent immediate reoperation and the EDH was successfully evacuated.

The occurrence of a remote-site EDH after a decompressive craniectomy is a rare but well-described

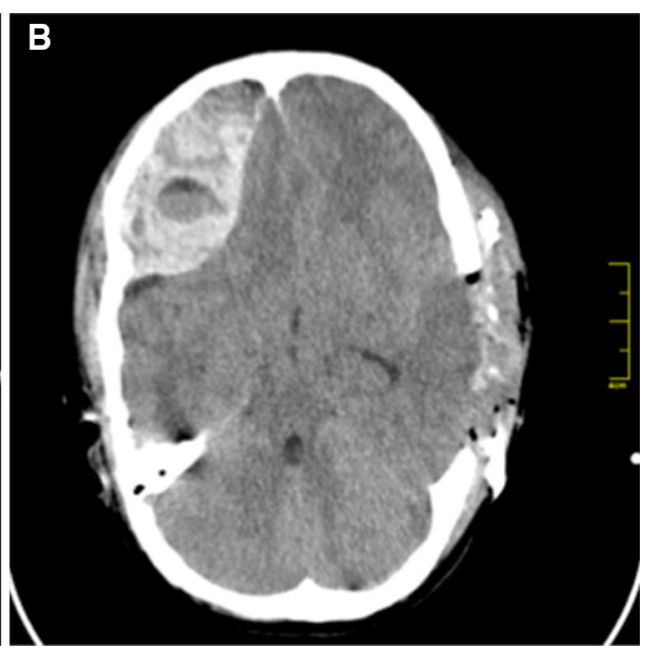

demonstrates a large right frontotemporal epidural hematoma with signs of diffuse intracranial hypertension and midbrain compression

Y. Aissaoui, MD $(\varangle) \cdot$ A. Belhadj, MD

Department of Anesthesiology, Avicenna Military Hospital,

Cadi Ayyad University, Marrakesh, Morocco

e-mail: younes.aissaoui@live.fr 
complication. One of the proposed pathophysiological mechanisms suggests that the initial head trauma damages the meningeal vessels, but the initial bleeding is contained by a tamponade effect due to cerebral edema and high intracranial pressure. The craniectomy alleviates this tamponade effect by lowering the intracranial pressure. This in turn promotes bleeding from the damaged vessels and leads to the development of an EDH. Moreover, the dura mater in children and young adults is less adherent to the skull, favouring the expansion of such hematomas. The presence of a contralateral skull fracture, "wrong-side" mydriasis, postoperative neurological deterioration, and higher than expected intracranial pressure are all signs associated with this complication. Although a decompressive craniectomy is a straightforward surgical procedure, when clinicians perform the surgery, they must be aware of the existence of such rare but potentially lifethreatening complications in order to offer prompt diagnosis and appropriate treatment.

Conflicts of interest None declared. 